\title{
Long noncoding RNA UCA1 promotes anaplastic thyroid cancer cell proliferation via miR-135a-mediated c-myc activation
}

\author{
YONGHUI WANG ${ }^{1}$, ZHENGGUANG HOU ${ }^{2}$ and DONG $\mathrm{LI}^{3}$ \\ ${ }^{1}$ Department of Breast Surgery, Weifang People's Hospital, Weifang, Shandong 261041; \\ ${ }^{2}$ Department of Breast Surgery, Linyi Central Hospital, Linyi, Shandong 276400; \\ ${ }^{3}$ Department of No. 1 Endocrinology, Affiliated Hospital of Jining Medical University, Jining, Shandong 272029, P.R. China
}

Received November 5, 2017; Accepted May 8, 2018

DOI: $10.3892 / \mathrm{mmr} .2018 .9276$

\begin{abstract}
The long non-coding RNA (lncRNA), urothelial carcinoma-associated 1 (UCA1), has been demonstrated to be dysregulated and serves a role in the progression of several cancer types. However, the exact effects and underlying molecular mechanisms of UCA1 in anaplastic thyroid cancer (ATC) remain unknown. The aim of the present study was to investigate the detailed function and the mechanism of UCA1 in the regulation of ATC cell progression. The present study identified that the expression levels of UCA1, in ATC cell lines and tissues, were significantly upregulated compared with normal human thyroid cell line and adjacent non-cancerous tissues, respectively. UCA1 knockdown significantly inhibited ATC cell viability, proliferation, migration and invasion and the expression level of c-myc proto-oncogene (c-myc) in vitro, and suppressed ATC tumor growth in vivo. In addition, using luciferase assays, it was confirmed that miR-135a directly bound to UCA1 and the 3' untranslated region of c-myc, and UCA1 competed with c-myc for miR-135a binding. miR-135a inhibition may upregulate c-myc expression, however, the upregulation of c-myc may be partially reduced by short hairpin UCA1. The present results illustrated that UCA1 promoted ATC cell proliferation through acting as a competing endogenous RNA by binding miR-135a. In conclusion, in the present study, UCA1 served as an oncogenic long non-coding RNA promoting ATC cell proliferation and may be a potential target for human ATC treatment.
\end{abstract}

\section{Introduction}

Thyroid cancer (TC) accounts for only $1 \%$ of all malignancies and is a common endocrine cancer that derives from

Correspondence to: $\mathrm{Dr}$ Dong Li, Department of No. 1 Endocrinology, Affiliated Hospital of Jining Medical University, 89 Guhuai Road, Jining, Shandong 272029, P.R. China

E-mail: 544471151@qq.com

Key words: anaplastic thyroid cancer, long non-coding RNA, urothelial carcinoma-associated 1, microRNA-135a, c-myc follicular thyroid or parafollicular C cells (1). In the USA, TC incidence is rapidly increasing with an estimated number of 56,870 individuals diagnosed with TC and an annual total mortality of 2,010 cases in 2017 (2). TCs are typically classified as papillary, follicular and anaplastic carcinomas (3). Anaplastic thyroid cancer (ATC) accounts for 1-2\% of all thyroid tumors and is characterized by aggressive, local invasion and common distant metastases (3). Currently, available therapy for ATCs includes chemotherapy, radiotherapy and surgery (3). However, no effective target treatments are available. ATC is one of the most fatal cancer types, with a mean survival of 6 months $(3,4)$. Therefore, an improved understanding of the molecular mechanisms underlying carcinogenesis and progression in ATC may contribute to the identification novel diagnostic markers and novel therapeutic targets.

It is well known that $>90 \%$ of the human genome is actively transcribed, but only $\sim 2 \%$ of the genome encodes proteins (5). The rest of the genome encodes non-coding RNAs (ncRNAs) including microRNAs (miRs) and long non-coding RNAs (lncRNAs). lncRNAs are defined as transcripts of $>200$ nucleotides in length with minimal to no protein-coding function (6). IncRNAs have been defined as a novel class of regulatory factors that modulate gene expression in physiological and pathological states (7). Their biological roles have been increasingly recognized; however, the functions of overexpressed lncRNAs in malignant diseases remain to be elucidated. UCA1, a sequence that consists of three exons with $1.4 \mathrm{~kb}$ in length, is a lncRNA originally identified in bladder transitional cell carcinoma (8) and promotes tumor progression in certain carcinomas. However, the expression and function of UCA1 in ATC has not yet been elucidated.

The present study demonstrated that UCA1 was significantly increased in ATC tissues and cell lines. Silencing of UCA1 inhibited ATC cell viability, proliferation, migration and invasion in vitro and inhibited the tumor growth in vivo. In addition, it was identified that c-myc proto-oncogene (c-myc) was a target gene of UCA1. UCA1 directly interacted with miR-135a and decreased the binding of miR-135a to the c-myc 3' untranslated region (UTR), which suppressed the degradation of c-myc mRNA by miR-135a. The results revealed that the UCA1/miR-135a/c-myc signaling pathway may be a potential novel therapeutic target for patients with ATC. 


\section{Materials and methods}

Cell culture and tissue collection. The human Nthy-ori3-1 cell line and ATC cell lines, SW1736 and KAT-18, were purchased from the American Type Culture Collection (Manassas, VA, USA). The cell lines were authenticated by short-tandem repeat profiling performing by BMR Genomics (Padova, Italy). The cells were cultured in Dulbecco's modified Eagle's medium (DMEM; HyClone; GE Healthcare Life Sciences, Logan, UT, USA) supplemented with $10 \%$ fetal bovine serum (FBS; HyClone; GE Healthcare Life Sciences) in a 95\% humidified atmosphere with $5 \% \mathrm{CO}_{2}$ at $37^{\circ} \mathrm{C}$. Human ATC specimens and their adjacent normal thyroid tissues (eight pairs) were collected from patients (three males and five females; aged 43-67 years) who underwent surgery, according to an approved human protocol at the Weifang People's Hospital from February 2016 to December 2016. The present study was approved by the Ethics Committee of Weifang People's Hospital (Weifang, China). Written informed consent was obtained from each patient.

Cell transfection. The LV3 (H1/GFP\&Puro) vector was synthesized to express the Lv-shRNA-UCA1 (Guangzhou Ribobio, Co., Ltd., Guangzhou, China). A non-target scrambled oligonucleotide served as the negative control (NC; Guangzhou RiboBio Co., Ltd., Guangzhou, China). The short hairpin RNA (shRNA) sequences used in the present study were as follows; shUCA1: 5'-GCCACCUACAUUAAAGCU AdTdT-3' and sh-control: 5'-CAGUACUUUUGUGUAGUA CAA-3'. The SW1736 and KAT-18 cells $\left(1 \times 10^{4}\right.$ cells/well) were grown in 6-well plates until the cells reached $50 \%$ confluency. The medium was replaced with $1 \mathrm{ml}$ fresh culture medium supplemented with $100 \mu \mathrm{l}$ viral supernatant $\left(1 \times 10^{8}\right.$ transducing units $/ \mathrm{ml}$ ) and $8 \mu \mathrm{g} / \mathrm{ml}$ Polybrene (both Hanbio Biotechnology Co., Ltd., Shanghai, China) for $24 \mathrm{~h}$. The SW1736 and KAT-18 cells were further cultured in medium containing $3 \mu \mathrm{g} / \mathrm{ml}$ puromycin for five passages and subsequently used in further experiments. Individual puromycin-resistant colonies were isolated during drug screening. The miR-135a mimic, mimic control (miR-NC), miR-135a inhibitor (anti-miR-135a) and inhibitor control (anti-NC) were synthesized by Shanghai GeneChem Co., Ltd. (Shanghai, China). The sequences were as follows: miR-135a mimic: 5'-UAUGGCUUUUUAUUC CUAUGUGAAGCAUAGGAAUAAAAAGCCAUAUU-3'; mimic control: 5'-CAGUACUUUUGUGUAGUACAA-3'; miR-135a inhibitor: 5'-UCACAUAGGAAUAAAAAGCCA UA-3'; and inhibitor control: 5'-CAGUACUUUUGUGUAGUA CAA-3'. The SW1736 and KAT-18 Cells $\left(2 \times 10^{5}\right)$ were seeded into 6-well plates $24 \mathrm{~h}$ prior to transfection, and a final concentration of $50 \mathrm{nM}$ miR-135a mimic, miR-NC, anti-miR-135a and anti-NC was used for transfection. Transfection was performed using Lipofectamine ${ }^{\circledR} 2000$ reagent (Invitrogen; Thermo Fisher Scientific, Inc., Waltham, MA, USA) according to the manufacturer's protocol. Subsequently, transfection efficiencies were determined following $48 \mathrm{~h}$ using the quantitative polymerase chain reaction (qPCR) method.

Western blot analysis. The expression level of protein c-myc was analyzed by western blotting. SW1736 and KAT-18 cells were lysed to extract the total proteinusing radioimmunoprecipitation assay lysis buffer (Promega Corporation, Madison, WI, USA) according to the manufacturer's protocol. Protein concentration was determined using a bicinchoninic acid Protein Assay kit (Thermo Fisher Scientific, Inc.) according to the manufacturer's protocol. The same amount of protein $(40 \mu \mathrm{g})$ from each cell line was separated by $12 \%$ SDS-PAGE and transferred onto polyvinylidene difluoride membranes. The membranes were blocked using $3 \%$ non-fat milk for $30 \mathrm{~min}$ at $4^{\circ} \mathrm{C}$. The membranes were subsequently respectively incubated with various diluted primary antibodies against c-myc (1:500; cat. no. 5605; Cell Signaling Technology, Inc., Danvers, MA, USA) and mouse monoclonal GAPDH (1:500; cat. no. AG019; Beyotime Institute of Biotechnology, Haimen, China). Following incubation overnight at $4^{\circ} \mathrm{C}$, horseradish peroxidase-conjugated goat anti-rabbit secondary antibody (1:2,000; cat. no. A0216; Beyotime Institute of Biotechnology) was added and incubated at room temperature for $2 \mathrm{~h}$. Specific bands were visualized with an enhanced chemiluminescent reagent (Nanjing KeyGen Biotech Co., Ltd., Nanjing, China) on an autoradiographic film. For quantitative assay, images were analyzed using ImageJ software (version $1.48 \mathrm{u}$; National Institutes of Health, Bethesda, MD, USA).

Reverse transcription ( $R T)$ - $q P C R$. Total RNA from cells was extracted using TRIzol ${ }^{\circledR}$ reagent (Invitrogen; Thermo Fisher Scientific, Inc.) according to the manufacturer's protocol. Complementary (c)DNA was synthesized from $500 \mathrm{ng}$ total RNA, and a reaction mixture $(20 \mu \mathrm{l})$ containing $1 \mu \mathrm{g}$ total RNA was reverse transcribed to cDNA using a PrimeScript RT Reagent kit with gDNA Eraser (Takara Biotechnology Co., Ltd., Dalian, China) according to the manufacturer's protocol. qPCR was performed using a SYBR Premix Ex Taq (Takara Biotechnology Co., Ltd.). The following conditions were applied for detecting mRNAs: $95^{\circ} \mathrm{C}$ for $30 \mathrm{sec}$; followed by 40 cycles of $95^{\circ} \mathrm{C}$ for $30 \mathrm{sec} ; 60^{\circ} \mathrm{C}$ for $30 \mathrm{sec}$; and $72^{\circ} \mathrm{C}$ for $30 \mathrm{sec}$. GAPDH was used as an internal control for the detection of c-myc and UCA1. U6 was used as an internal control for the detection of miR-135a. The relative mRNA expression levels were calculated as the inverse log of $\Delta \Delta \mathrm{Cq}$ and normalized to the reference (9). The primer sequences were as follows: UCA1, forward 5'-TTTGCCAGCCTC AGCTTAAT-3' and reverse 5'-TTGTCCCCATTTTCCATC AT-3'; miR-135a, forward 5'-GCGCGTATGGCTTTTTAT TCCT-3' and reverse 5'-CAGTGCAGGGTCCGAGGTC-3'; U6, forward 5'-TGCGGGTGCTCGCTTCGCAGC-3' and reverse 5'-CCAGTGCAGGGTCCGAGGT-3'; c-myc, forward 5'-TCAAGAGGCGAACACACAAC-3' and reverse 5'-GGC CTTTTCATTGTTTTCCA-3'; GAPDH, forward 5'-CTG ACCTGCCGTCTAGAAA-3' and reverse 5'-GTGGTGTGA CTTAGAGGGG-3'.

Cell viability assay. Cell viability was measured using a Cell Counting Kit-8 (CCK-8) assay (Beyotime Institute of Biotechnology). SW1736 and KAT-18 cells were plated onto 96-well plates at a density of 3,000 cells/well. Following culture at the indicated time points $(0,24,48$ and $72 \mathrm{~h}), 10 \mu \mathrm{l}$ CCK-8 solution was added into each well at $37^{\circ} \mathrm{C}$. After $3 \mathrm{~h}$, the absorbance of each well was measured using a Multiskan MK3 device (Thermo Fisher Scientific, Inc.) at a wavelength of $450 \mathrm{~nm}$. 
A

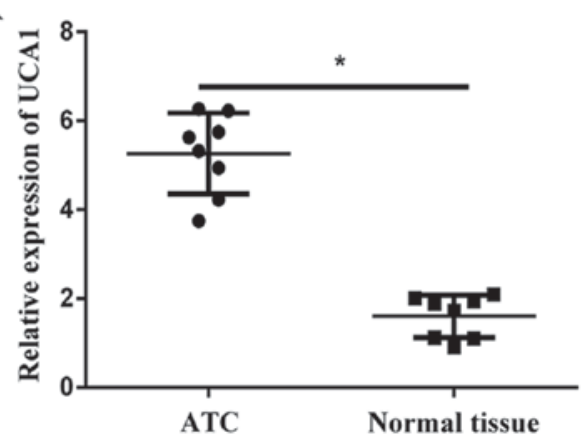

B

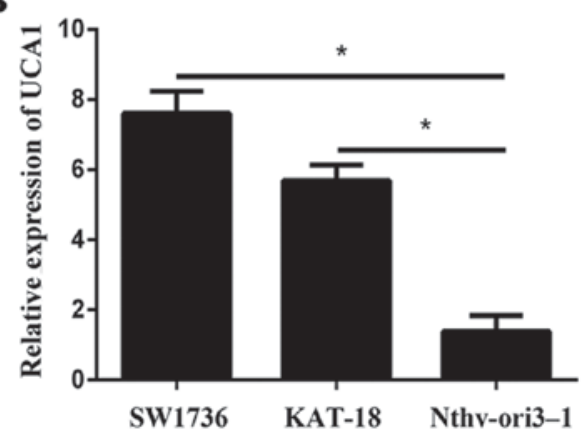

Figure 1. UCA1 expression was upregulated in ATC tissues and cell lines. (A) Relative UCA1 expression levels were analyzed by reverse transcription-quantitative polymerase chain reaction in 8 pairs of anaplastic thyroid cancer specimens and adjacent normal tissues. GAPDH was used as internal control. ${ }^{*} \mathrm{P}<0.05$. (B). Relative expression level of UCA1 was examined in ATC cell lines (SW1736 and KAT-18) and in the normal human follicular thyroid cells Nthy-ori3-1. GAPDH was used as internal control. "P<0.05. UCA1, urothelial carcinoma-associated 1; ATC, anaplastic thyroid cancer.

Colony formation assay. The cells (at a density of 400 cells/well) transfected with shUCA1 and sh-control were placed into 6-well plates. After 1 week of culture, the colonies were fixed with $10 \%$ methanol for $15 \mathrm{~min}$ at room temperature and stained with $0.1 \%$ crystal violet (Beyotime Institute of Biotechnology) for $20 \mathrm{~min}$ at room temperature. Colonies with $>50$ cells were counted using the CKX41 light microscope (magnification, x100; Olympus Corporation, Tokyo, Japan).

Cell migration and invasion assay. Cell migration and invasion were assessed by performing a Transwell assay (Corning Incorporated, Corning, NY, USA). For the invasion assay, the upper chambers were coated with $50 \mu$ l Matrigel (BD Biosciences, Franklin Lakes, NJ, USA). Cells were harvested at $48 \mathrm{~h}$ post-transfection. A total of $5 \times 10^{4}$ cells in $200 \mu \mathrm{l}$ DMEM (HyClone; GE Healthcare Life Sciences) were seeded in the upper chamber. The lower chamber was filled with DMEM supplemented with 5\% FBS. Following incubation at $37^{\circ} \mathrm{C}$ with $5 \% \mathrm{CO}_{2}$ for $8 \mathrm{~h}$ (migration) or $12 \mathrm{~h}$ (invasion), the cells on the lower chamber were fixed with $10 \%$ methanol for $15 \mathrm{~min}$ at room temperature and stained with crystal violet for $20 \mathrm{~min}$ at room temperature. The number of the colonies that had migrated through the pores was quantified by randomly counting 10 independent visual fields using the CKX41 light microscope (magnification, x200; Olympus Corporation, Tokyo, Japan).

Bioinformatics analysis. Targetscan software (http://www. targetscan.org/vert 72/) was used to predict the possible targets of miR-135a by searching miR-135a. The putative miRNA binding sites on UCA1 sequences were predicted by an RNAhybrid software program (http://bibiserv2.cebitec. uni-bielefeld.de/rnahybrid) with the minimum free energy cutoff set at $22 \mathrm{kcal} / \mathrm{mol}$.

Luciferase reporter assay. The binding site of $\mathrm{miR}-135 \mathrm{a}$ in the 3'-UTR of the target mRNA was cloned into the pmirGLO Dual-Luciferase miRNA Target Expression Vector (Promega Corporation), according to the manufacturer's protocol. SW1736 and KAT-18 cells $\left(4 \times 10^{5}\right)$ cultured in 24-well plates were co-transfected with luciferase reporter plasmids [wild type (wt)-c-myc, mutant (mut)-c-myc containing miR-135a binding site, wt-UCA1 and mut-UCA1 containing miR-135a binding site] and miR-135a mimics or miR-135a inhibitor (Shanghai GeneChem Co., Ltd., Shanghai, China) using Lipofectamine ${ }^{\circledR} 2000$ (Invitrogen; Thermo Fisher Scientific, Inc.). The sequences were as follows; miR-135a mimic: 5'-UAUGGCUUUUUAUUCCUAUGUGAAGCAUAGGAAUAA AAAGCCAUAUU-3' and miR-135a inhibitor: 5'-UCACAU AGGAAUAAAAAGCCAUA-3'. After 48 h co-transfection, the luciferase activity was measured using a dual-luciferase reporter assay system (Promega Corporation, Madison, WI, USA) according to the manufacturer's protocol. The data was normalized to Renilla luciferase activity.

Animal studies. All experiments involving animals were approved by the Animal Care and Welfare Committee of Weifang People Hospital. SW1736 cells $\left(1 \times 10^{6}\right)$ transfected with sh-control or sh-UCA1 were subcutaneously injected into the right flanks of four-week-old BALB/c athymic nude mice ( $n=12$; female; weight range, 20-22 g; Yangzhou University, Yangzhou, China) and maintained in a specific pathogen free environment with constant humidity (45-50\%) and constant temperature $\left(25-27^{\circ} \mathrm{C}\right)$ under a $12 \mathrm{~h}$ light/dark cycle with free access to food and water. Tumor volume $\left(\mathrm{mm}^{3}\right)$ was calculated every 3 days for 3 weeks using the formula $V=0.5 x$ length $\mathrm{x}$ width ${ }^{2}$. Tumors were collected and images were captured 3 weeks after inoculation.

Statistical analysis. All experiments were performed in triplicate. Unless otherwise indicated, the data were presented as the mean \pm standard deviation. Statistical significance was determined using an unpaired Student's t-test or one-way analysis of variance with Tukey's test as the post hoc test using SPSS software version 13.0 (SPSS, Inc., Chicago, IL, USA). $\mathrm{P}<0.05$ was considered to indicate a statistically significant difference.

\section{Results}

UCA1 is upregulated in ATC tissues and cell lines. Initially the expression levels of UCA1 in eight paired samples (ATC specimens and corresponding adjacent normal tissues) were examined using RT-qPCR. Results revealed that UCA1 expression was significantly higher in tumor tissues compared with adjacent normal tissues (Fig. 1A). In addition, the expression 
A
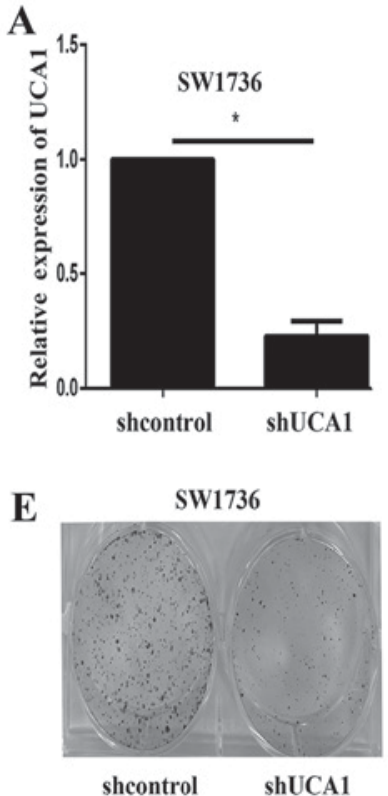

G

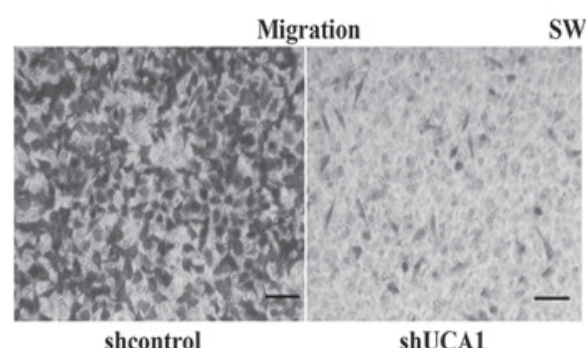

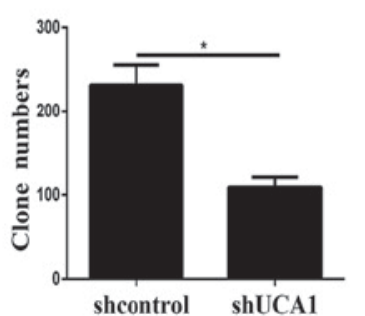

C

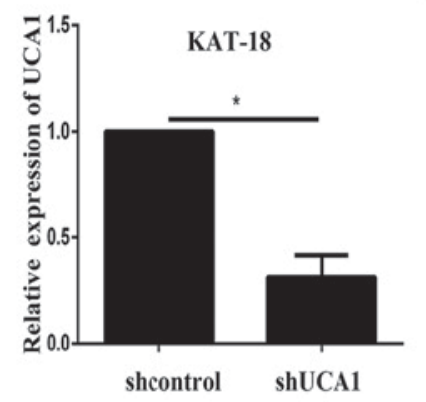

F

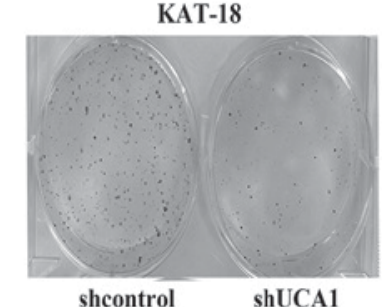

Invasion

KAT-18
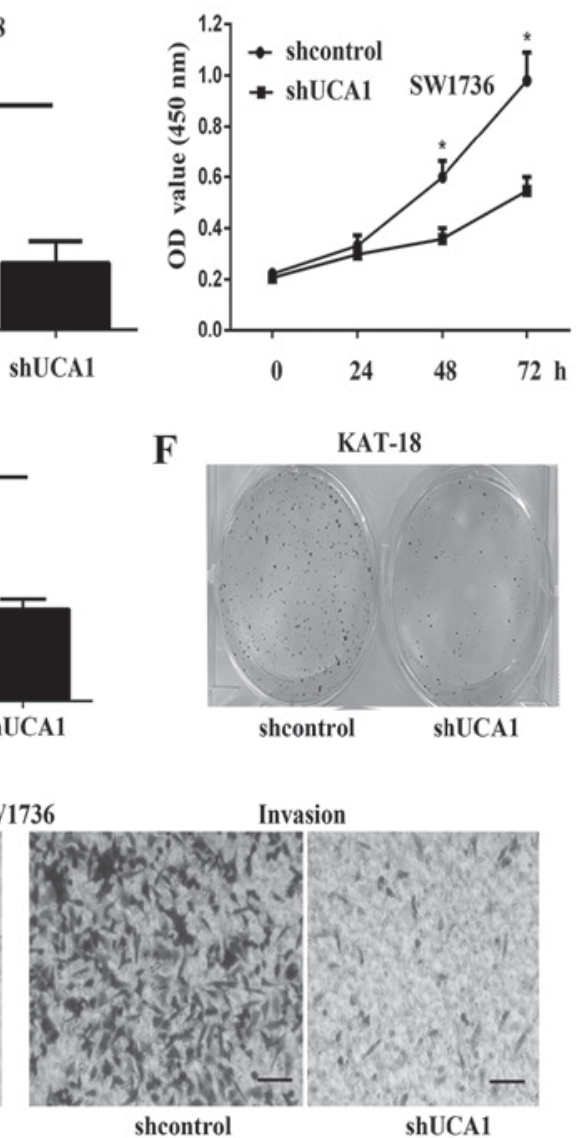

Invasion

knt.

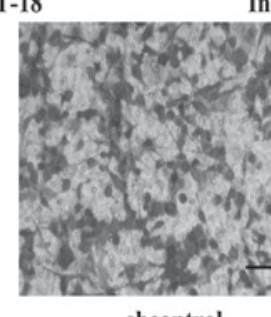

shcontrol

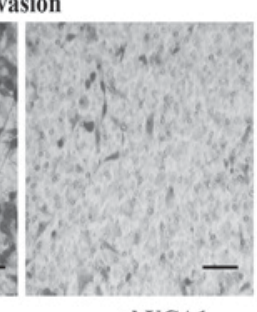

shUCA1
D
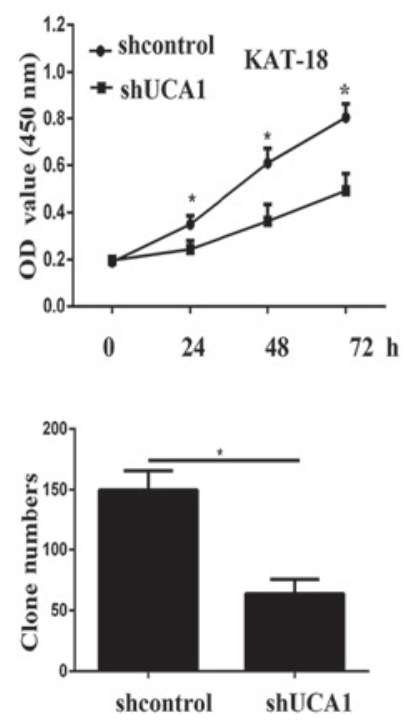

H

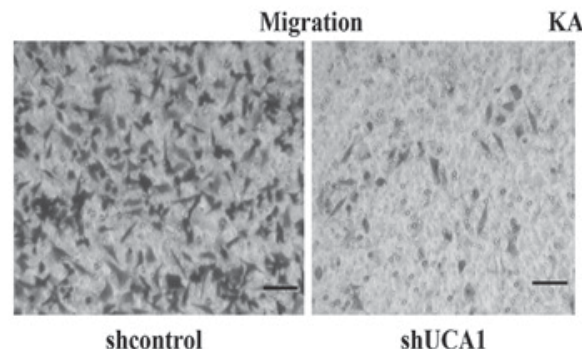

Figure 2. UCA1 promoted ATC cell proliferation. (A) SW1736 and (B) KAT-18 were transfected with sh-UCA1 and sh-control, then the expression levels of UCA1 were determined by reverse transcription-quantitative polymerase chain reaction. Cell viability and proliferation were separately assessed by Cell Counting kit-8 in (C) SW1736 and (D) KAT-18 cells. Colony formation assays were preformed using (E) SW1736 and (F) KAT-18 cells. Representative images of migration and invasion of (G) SW1736 and (H) KAT-18 cells transfected with shUCA1 and shcontrol were shown on the left panel (magnification, x200; scale bar, $50 \mu \mathrm{m}$ ). The number of migrated and invaded cells was measured in the right panel graphs. ${ }^{\text {P }}<0.05$. UCA1, urothelial carcinoma-associated 1 ; ATC, anaplastic thyroid cancer; sh, short hairpin.

of UCA1 in ATC cell lines (SW1736 and KAT-18) was significantly increased compared with the normal human follicular thyroid cell Nthy-ori3-1 (Fig. 1B).

UCA1 silencing suppresses cell viability, proliferation, migration and invasion in vitro. Partial UCA1 knockdown was achieved by transfection with shUCA1 in SW1736 and KAT-18, as verified using RT-qPCR (Fig. 2A and B). As demonstrated by CCK-8 assays, the cell viability of SW1736 and KAT-18 cells was significantly inhibited after UCA1 knockdown (Fig. 2C and D). In addition, the colony formation assay indicated that the proliferation of UCA1 knockdown cells was inhibited (Fig. 2E and F). The Transwell assay indicated that UCA1 knockdown significantly inhibited cell migration and invasion (Fig. 2G and H).
miR-135a negatively regulates $c$-myc expression. To further investigate the underlying mechanism by which UCA1 regulates ATC progression, Targetscan 7.2 (http://www.targetscan. org/vert 72/) was used. A previous study has illustrated that c-myc is involved in the progression of various tumors (10). c-myc silencing was demonstrated to decrease the tumor viability, proliferation and migration in vitro and in vivo (10). c-myc was predicted to be a target of miR-135a. SW1736 and KAT-18 cells were transfected with miR-135a mimic, miR-NC, anti-miR-135a or anti-NC. RT-qPCR results demonstrated the expression level of miR-135a in SW1736 and KAT-18 cell lines transfected with with miR-135a mimic, miR-NC, anti-miR-135a and anti-NC (Fig. 3A and B). miR-135a mimic significantly increased the expression of miR-135a in SW1736 
A

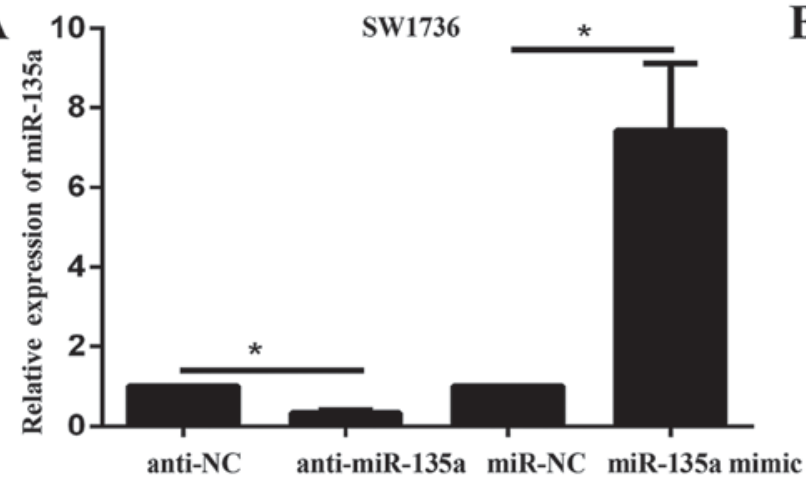

B

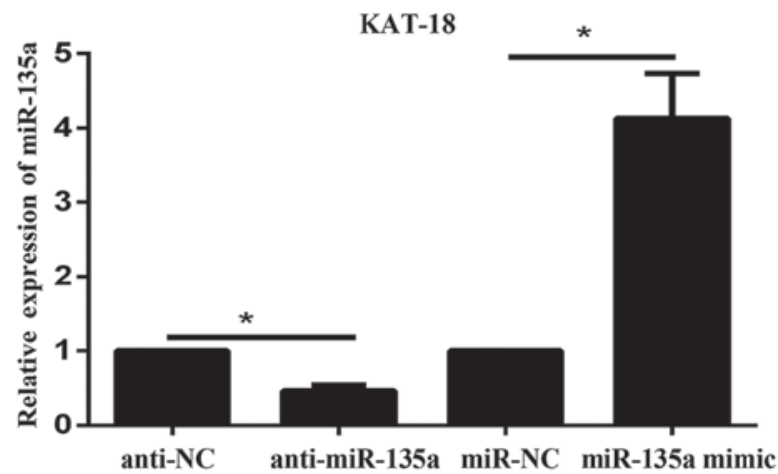

C

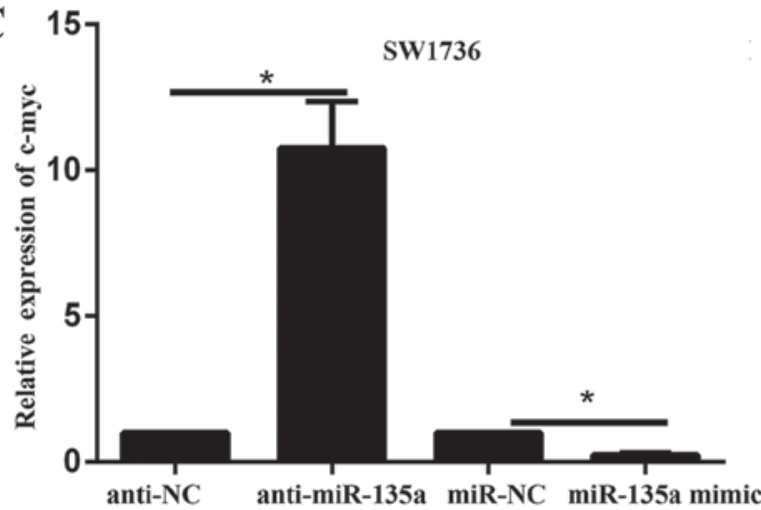

D

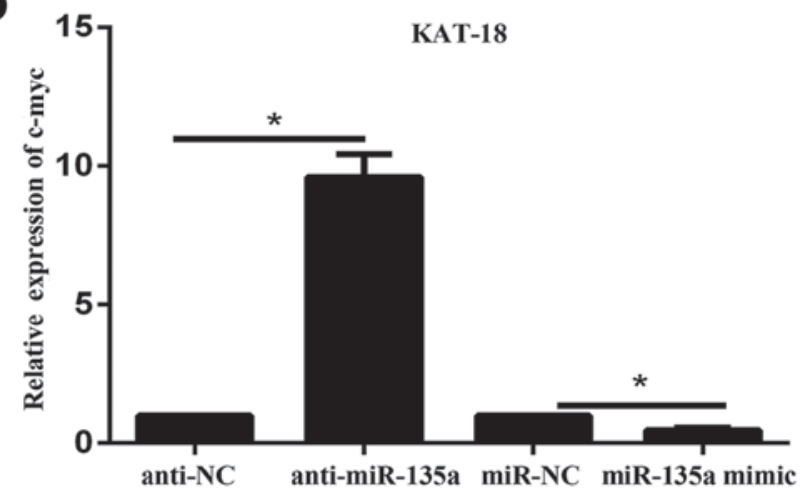

$\mathbf{E}$

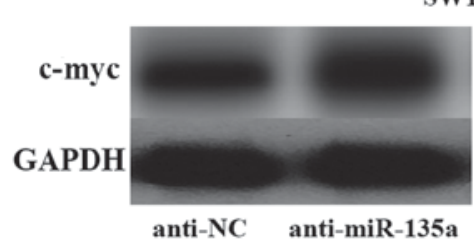

SW1736

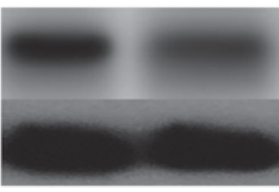

miR-NC miR-135a mimic

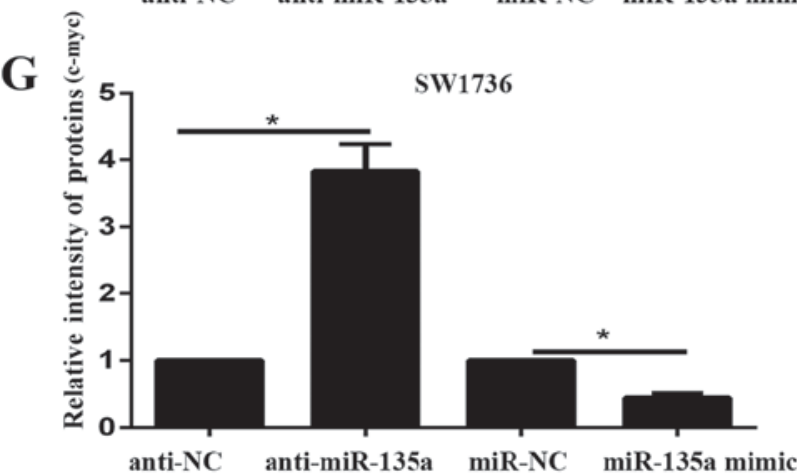

$\mathbf{F}$

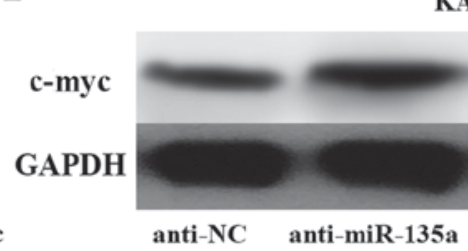

KAT-18

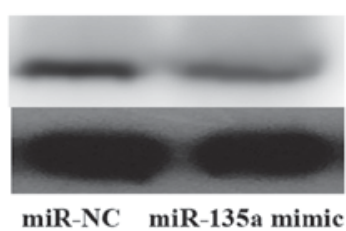

$\mathbf{H}$

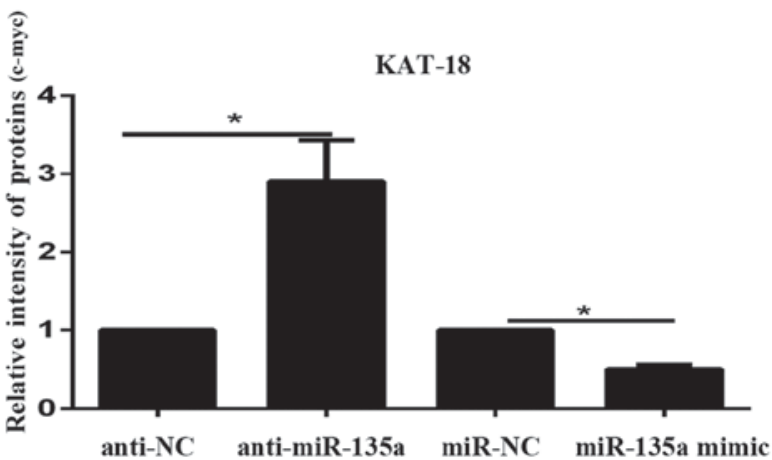

Figure 3. miR-135a may negatively regulate c-myc expression. Relative expression levels of miR-135a were determined in (A) SW1736 and (B) KAT-18 cells transfected with miR-135a mimic, miRNA NC, anti-miR-135a or anti-NC by RT-qPCR. mRNA expression levels of c-myc in (C) SW1736 and (D) KAT-18 cells in response to miR-135a expression were determined using RT-qPCR. Western blotting was used to determine c-myc expression levels in (E) SW1736 and (F) KAT-18 cells. Protein expression levels were quantitatively analyzed in (G) SW1736 and (H) KAT-18 cells. "P<0.05. miR, microRNA; NC, negative control; ATC, anaplastic thyroid cancer; c-myc, c-myc proto-oncogene; anti-miR-135a, miR-135a inhibitor; anti-NC, negative control inhibitor; RT-qPCR, reverse transcription-quantitative polymerase chain reaction.

and KAT-18 cells; in contrast, anti-miR-135a significantly decreased the expression of miR-135a in the two cell lines $(\mathrm{P}<0.05)$. The mRNA and protein expression levels of c-myc in SW1736 and KAT-18 cell lines in response to miR-135a overexpression or inhibition were subsequently examined via RT-qPCR and western blotting. The results indicated that the mRNA and protein expression of c-myc decreased following miR-135a overexpression and increased by miR-135a inhibition (Fig. 3C-H). These data suggested that miR-135a may negatively regulate c-myc expression in ATC cell lines.

UCA1 competes with c-myc for miR-135a binding. The present study demonstrated that miR-135a negatively regulated c-myc expression. The association between UCA1 and miR-135a was subsequently determined. The expression level of UCA1 in response to miR-135a inhibition and overexpression in 
A

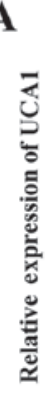

SW1736

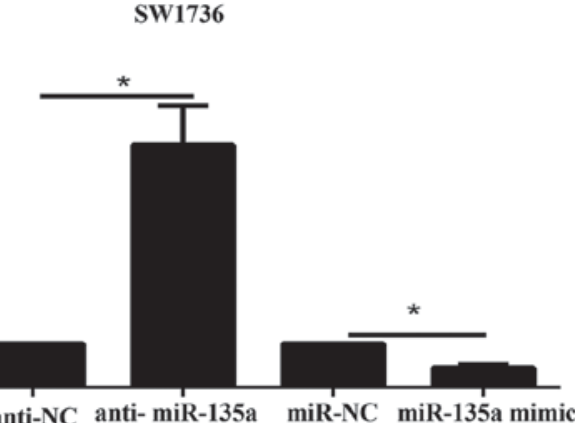

C

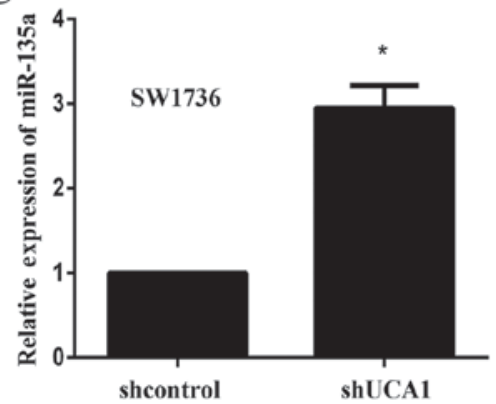

D

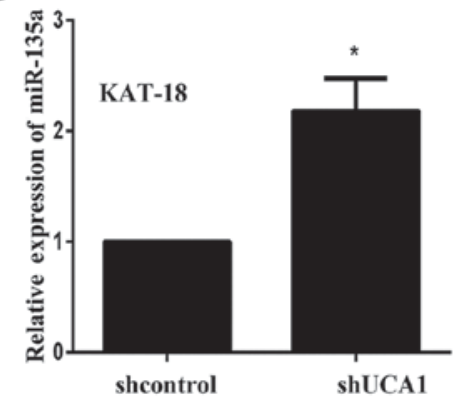

B

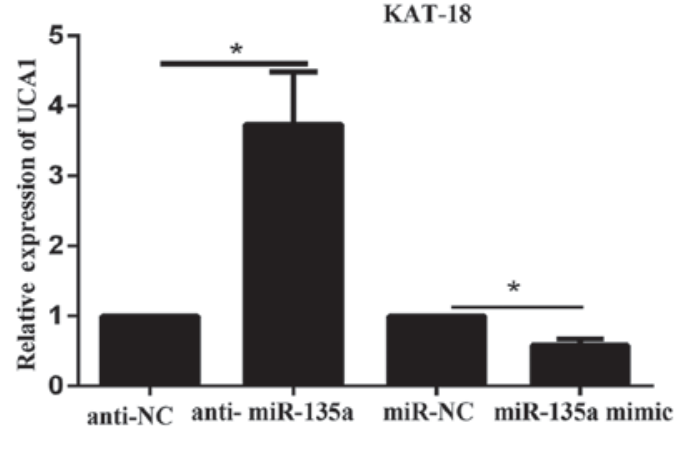

E
F

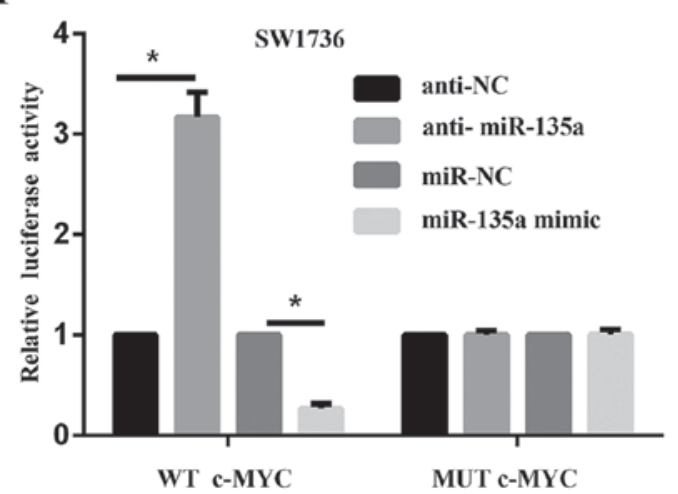

G

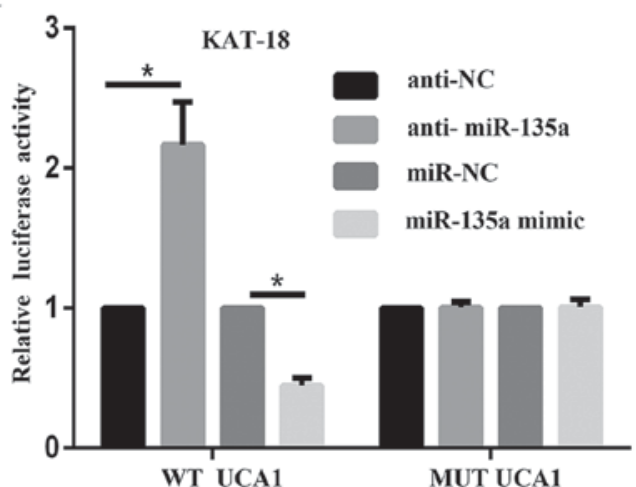

Figure 4. UCA1 competes with c-myc for miR-135a binding. UCA1 expression in response to miR-135a overexpression and inhibition in (A) SW1736 and (B) KAT-18 cells was examined using RT-qPCR. *P<0.05. miR-135a expression in response to UCA1 knockdown in (C) SW1736 and (D) KAT-18 cells was determined by RT-qPCR. "P<0.05 vs. the sh-control group. (E) Wt and mut UCA1 containing 5 bp mutation in the predicted binding sites of miR-135a, or c-myc 3'UTR (wt-c-myc 3'UTR and mut-c-myc 3'UTR containing a 5 bp mutation in the predicted binding sites of miR-135a) luciferase reporter gene vectors were constructed. After culturing overnight, (F) SW1736 and (G) KAT-18 cells were co-transfected with the indicated vectors and miR-135a mimics or anti-miR-135a. Luciferase assays were performed $48 \mathrm{~h}$ after transfection using the Dual Luciferase Reporter Assay system to determine the luciferase activity. ${ }^{*} \mathrm{P}<0.05$. Wt, wild type; mut, mutant; UCA1, urothelial carcinoma-associated 1; miR, microRNA; 3'UTR, 3' untranslated region; c-myc, c-myc proto-oncogene; RT-qPCR, reverse transcription-quantitative polymerase chain reaction; anti-miR-135a, miR-135a inhibitor.

SW1736 and KAT-18 cells was determined via RT-qPCR. The results illustrated that UCA1 decreased following miR-135a overexpression and increased by miR-135a inhibition (Fig. 4A and B). Furthermore, RT-qPCR results indicated that miR-135a expression was significantly increased following UCA1 silencing (Fig. 4C and D). These data suggested a dual regulation between UCA1 and miR-135a. According to online tools, UCA1 shared the same binding site in miR-135a with c-myc (Fig. 4E). Luciferase assays were performed to determine the association between miR-135a and UCA1. Luciferase reporter gene vectors were constructed and co-transfected into SW1736 and KAT-18 cells with miR-135a mimic or anti-miR-135a. The luciferase activity was monitored using dual luciferase assays. The results indicated that the luciferase activity of wt-UCA1 and wt-c-myc vectors was significantly decreased by miR-135a mimics and increased by the miR-135a inhibitor. Following mutation in the predicted binding sites of miR-135a, the alterations in the luciferase activity were abolished (Fig. 4F and G). These data suggested that UCA1 and c-myc may bind to miR-135a, and UCA1 may compete with c-myc for miR-135a binding.

UCA1 regulates c-myc expression via miR-135a. The present study measured the protein expression levels of c-myc in SW1736 and KAT-18 cells, in response to UCA1 silencing. The RT-qPCR and western blotting results indicated that UCA1 knockdown significantly decreased the expression of c-myc at the mRNA and protein level, respectively (Fig. 5). These results suggested that UCA1 may regulate tumor progression through c-myc. To validate whether UCA1 regulated c-myc expression through miR-135a, the present study co-transfected SW1736 and KAT-18 cells with the miR-135a inhibitor and 
A
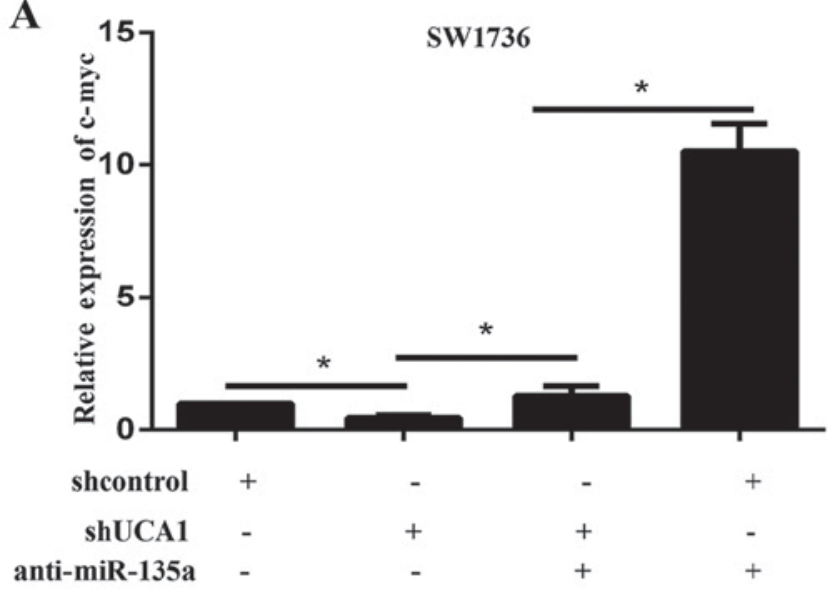

C

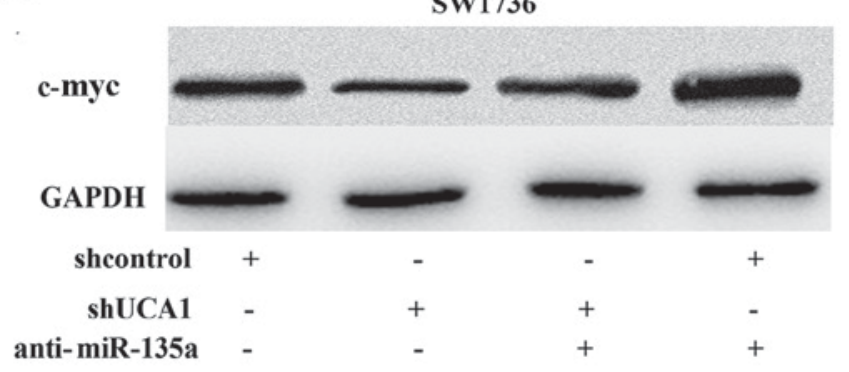

B
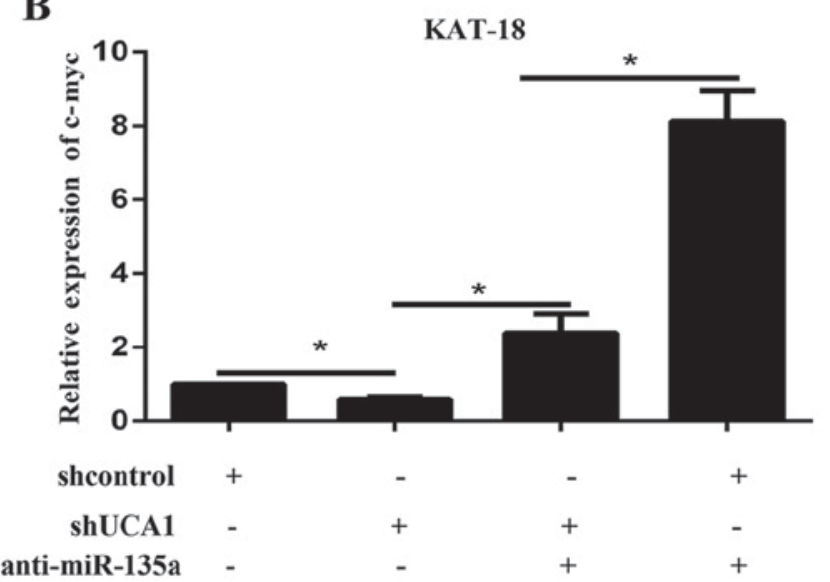

D

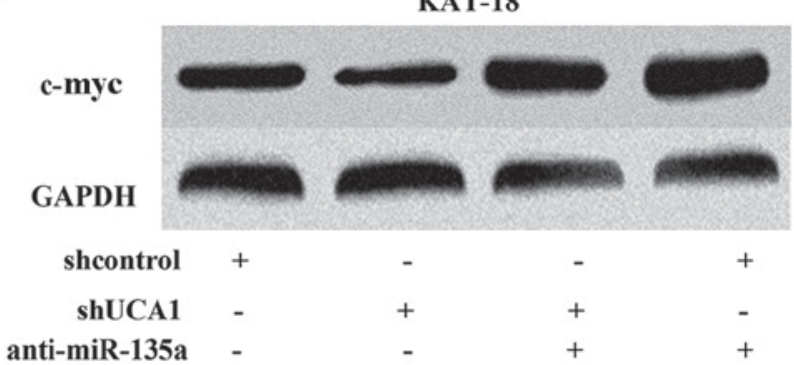

Figure 5. UCA1 regulates c-myc expression via miR-135a. Cells were co-transfected with sh-UCA1 and anti-miR-135a, and the mRNA and protein expression levels of c-myc were measured via reverse transcription-quantitative polymerase chain reaction in (A) SW1736 and (B) KAT-18 cells, and western blotting in (C) SW1736 and (D) KAT-18 cells. * P<0.05. UCA1, urothelial carcinoma-associated 1; miR, microRNA; c-myc, c-myc proto-oncogene; anti-miR-135a, miR-135a inhibitor.

shUCA1, and examined the expression of c-myc by qPCR and western blot. The results demonstrated that the mRNA and protein expression levels of c-myc were increased by miR-135a inhibition and reduced by shUCA1 (Fig. 5A-D). The promotive effect of the miR-135a inhibitor on the expression of c-myc may be partially abolished by shUCA1 (Fig. 5A-D). These data suggested that UCA1 regulated c-myc expression through miR-135a.

Knockdown of UCA1 inhibits tumor growth in vivo. To further illustrate the effects of UCA1 on ATC growth in vivo, the present study used a xenograft model in which SW1736 cells transfected with shUCA1 or sh-NC were subcutaneously injected into the flanks of athymic mice and were allowed to develop measurable tumors. The results demonstrated that tumors formed by transfected shUCA1 grew more slowly compared with those formed by transfected sh-NC (Fig. 6A-C). Furthermore, RT-qPCR indicated that the c-myc expression levels in the shUCA1 tumor xenografts were lower compared with those in the control xenografts (Fig. 6D). In addition, the RT-qPCR results indicated that the expression level of miR-135a was significantly downregulated in shUCA1-transfected tumors compared with sh-NC-transfected tumors (Fig. 6E).

\section{Discussion}

The present study demonstrated the oncogenic role of UCA1 in the progression of ATC. It was revealed that expression levels of UCA1 significantly increased in ATC cell lines and tissues. The present study also indicated that UCA1 knockdown significantly inhibited cell viability, proliferation, migration and invasion in ATC in vitro and in vivo. Finally, it was predicted that UCA1 binds to miR-135a and modulates c-myc expression.

LncRNAs are transcribed RNA molecules that are $>200$ nucleotides in length and lack significant protein-coding potential, however, they may regulate protein-coding genes at epigenetic, transcriptional and post-transcriptional levels, and serve a critical role in physiological processes (11). Previous studies have demonstrated that a variety of lncRNAs are frequently aberrantly expressed in cancer, and the differential expression of lncRNAs is closely associated with tumor progression, serving roles of oncogenes or tumor suppressor genes (12). To date, only a number of lncRNAs, including lncRNA LINC00312, have been demonstrated to be involved in TC development and progression (13). UCA1 is a recently identified lncRNA (8). Previous studies have identified that UCA1 promoted tumor progression in a wide range of tumor types, including bladder cancer, breast cancer, hepatocellular carcinoma, colorectal cancer and gastric cancer (14-18). However, the role and mechanism of UCA1 in ATC has not been illustrated. The present study initially demonstrated that UCA1 expression was significantly increased in ATC tissues and cell lines. The data suggested that UCA1 served as an oncogene in ATC, consistent with previous studies in the other tumor types $(8,14-18)$. A previous study illustrated that UCA1 regulated the growth and metastasis of pancreatic cancer by 
A

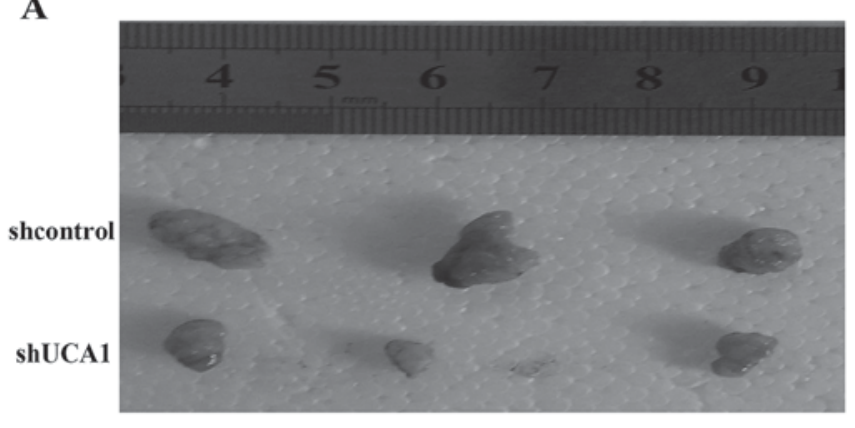

B

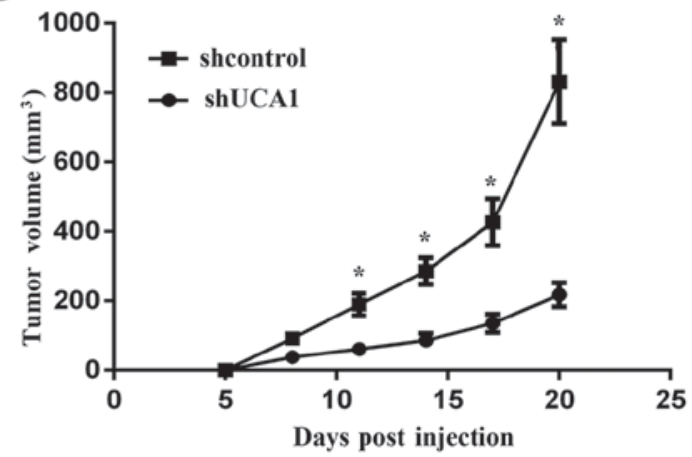

$\mathbf{E}$
C

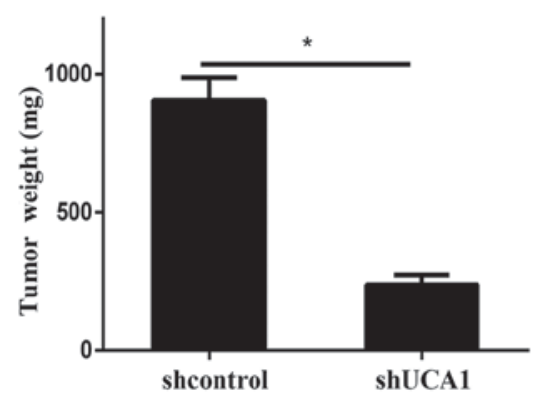

D

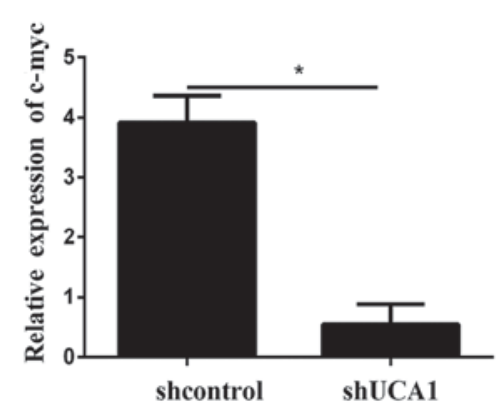

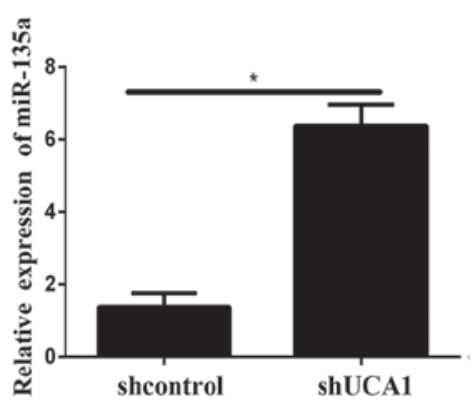

Figure 6. UCA1 silencing inhibits tumor growth in vivo. SW1736 cells transfected with shUCA1 or shNC were injected subcutaneously into athymic nude mice (n=6/group). (A) The shUCA1 tumor xenografts were smaller compared with the shNC tumor xenografts. (B) shUCA1 tumor growth was slower compared with the shNC tumor xenografts after 21 days. (C) The average weight of the resected tumors. (D) The expression level of c-myc in shUCA1 or shNC SW1736 tumors using RT-qPCR. (E) RT-qPCR analysis of miR-135a expression levels in the shUCA1 tumor xenografts and shNC tumor xenografts. ${ }^{*} \mathrm{P}<0.05$ vs. respective shUCA1. UCA1, urothelial carcinoma-associated 1; miR, microRNA; sh, short hairpin; NC, negative control; c-myc, c-myc proto-oncogene; RT-qPCR, reverse transcription-quantitative polymerase chain reaction.

binding to miR-135a (19). The present study illustrated the dual interaction of UCA1 and miR-135a in ATC, possibly for the first time. UCA1 and miR-135a may regulate each other in a negative way. To further demonstrate the underlying molecular mechanisms of the UCA1/miR-135a axis-induced cell proliferation in SW1736 and KAT-18 cells, the present study particularly focused on c-myc for further experiments.

The c-myc protein encodes a basic helix-loop-helix leucine zipper transcription factor and acts as an important regulator of several cellular processes, including protein synthesis, cell growth and proliferation in various cell types (20). Furthermore, the c-myc oncogene was overexpressed in a number of types of human cancer, including anaplastic thyroid cancer, and targeting of the c-myc protein may be a potential treatment modality for human ATC $(10,21)$. A previous study demonstrated that miR-135a inhibited cancer cell proliferation by targeting the c-myc oncogene in renal cell carcinoma (22). The present study measured the protein and mRNA expression levels of c-myc and UCA1. UCA1 knockdown reduced c-myc expression at the protein and mRNA level, indicating the involvement of c-myc in the regulation of UCA1 cell proliferation and invasion. Furthermore, by dual luciferase assays, the present study illustrated that UCA1 may bind to miR-135a, and miR-135a may directly bind to the 3'UTR of c-myc. The results suggested that UCA1 may compete with c-myc for miR-135a binding, and inhibit miR-135a and promote c-myc expression, to affect ATC progression.

In conclusion, the UCA $1 / \mathrm{miR}-135 \mathrm{a} / \mathrm{c}-\mathrm{myc}$ axis may serve a role in ATC cell proliferation and invasion, and may provide a potential therapeutic application in patients with ATC.

\section{Acknowledgements}

Not applicable.

\section{Funding}

The current study was supported by the Foundation of Shandong People and Family Planning Commission (grant no. 2015WSA07008).

\section{Availability of data and materials}

All data generated or analyzed during this study are included in this published article.

\section{Authors' contributions}

YW and DL conceived and designed the experiments. YW wrote and revised the manuscript. $\mathrm{ZH}$ conducted all experiments. All authors read and approved the final manuscript.

\section{Ethics approval and consent to participate}

The present study was approved by the ethics committee of Weifang People's Hospital (Weifang, China). All patients provided written informed consent. 


\section{Patient consent for publication}

All patients provided written informed consent for the publication of any associated data and accompanying images.

\section{Competing interests}

The authors declare that they have no competing interests.

\section{References}

1. Xing M: Molecular pathogenesis and mechanisms of thyroid cancer. Nat Rev Cancer 13: 184-199, 2013.

2. Siegel RL, Miller KD and Jemal A: Cancer statistics, 2017. CA Cancer J Clin 67: 7-30, 2017.

3. Nagaiah G, Hossain A, Mooney CJ, Parmentier J and Remick SC: Anaplastic thyroid cancer: A review of epidemiology, pathogenesis, and treatment. J Oncol 2011: 542358, 2011.

4. O'Neill JP and Shaha AR: Anaplastic thyroid cancer. Oral Oncol 49: 702-706, 2013

5. Ezkurdia I, Juan D, Rodriguez JM, Frankish A, Diekhans M, Harrow J, Vazquez J, Valencia A and Tress ML: Multiple evidence strands suggest that there may be as few as 19,000 human protein-coding genes. Hum Mol Genet 23: 5866-5878, 2014.

6. Geisler S and Coller J: RNA in unexpected places: Long non-coding RNA functions in diverse cellular contexts. Nat Rev Mol Cell Biol 14: 699-712, 2013.

7. Lee JT and Bartolomei MS: X-inactivation, imprinting, and long noncoding RNAs in health and disease. Cell 152: 1308-1323, 2013

8. Wang F,LiX,XieX,Zhao LandChen W: UCA1, a non-protein-coding RNA up-regulated in bladder carcinoma and embryo, influencing cell growth and promoting invasion. FEBS Lett 582: 1919-1927, 2008.

9. Livak KJ and Schmittgen TD: Analysis of relative gene expression data using real-time quantitative PCR and the 2(-Delta Delta C(T)) method. Methods 25: 402-408, 2001

10. Enomoto K, Zhu X, Park S, Zhao L, Zhu YJ, Willingham MC, Qi J, Copland JA, Meltzer P and Cheng SY: Targeting MYC as a therapeutic intervention for anaplastic thyroid cancer. J Clin Endocrinol Metab 102: 2268-2280, 2017.
11. Mercer TR and Mattick JS: Structure and function of long noncoding RNAs in epigenetic regulation. Nat Struct Mol Biol 20: 300-307, 2013.

12. Lin CY and Xu HM: Novel perspectives of long non-coding RNAs in esophageal carcinoma. Carcinogenesis 36: 1255-1262, 2015.

13. Liu K, Huang W, Yan DQ, Luo Q and Min X: Overexpression of long intergenic noncoding RNA LINC00312 inhibits the invasion and migration of thyroid cancer cells by down-regulating microRNA-197-3p. Biosci Rep 37: pii: BSR20170109, 2017.

14. Zhang S, Dong X, Ji T, Chen G and Shan L: Long non-coding RNA UCA1 promotes cell progression by acting as a competing endogenous RNA of ATF2 in prostate cancer. Am J Transl Res 9: 366-375, 2017.

15. Wang X, Yang B and Ma B: The UCA1/miR-204/Sirt1 axis modulates docetaxel sensitivity of prostate cancer cells. Cancer Chemother Pharmacol 78: 1025-1031, 2016.

16. Jiao C, Song Z, Chen J, Zhong J, Cai W, Tian S, Chen S, Yi Y and Xiao Y: IncRNA-UCA1 enhances cell proliferation through functioning as a ceRNA of Sox4 in esophageal cancer. Oncol Rep 36: 2960-2966, 2016.

17. Wang ZQ, Cai Q, Hu L, He CY, Li JF, Quan ZW, Liu BY, Li C and Zhu ZG: Long noncoding RNA UCA1 induced by SP1 promotes cell proliferation via recruiting EZH2 and activating AKT pathway in gastric cancer. Cell Death Dis 8: e2839, 2017.

18. Wang X, Gao Z, Liao J, Shang M, Li X, Yin L, Pu Y and Liu R: lncRNA UCA1 inhibits esophageal squamous-cell carcinoma growth by regulating the Wnt signaling pathway. J Toxicol Environ Health A 79: 407-418, 2016.

19. Zhang X, Gao F, Zhou L, Wang H, Shi G and Tan X: UCA1 regulates the growth and metastasis of pancreatic cancer by sponging MiR-135a. Oncol Res 25: 1529-1541, 2017.

20. Eilers M and Eisenman RN: Myc's broad reach. Genes Dev 22 2755-2766, 2008.

21. Pelengaris $\mathbf{S}$, Khan $\mathrm{M}$ and Evan G: c-MYC: More than just a matter of life and death. Nat Rev Cancer 2: 764-776, 2002.

22. Yamada Y, Hidaka H, Seki N, Yoshino H, Yamasaki T, Itesako T, Nakagawa $M$ and Enokida $H$ : Tumor-suppressive microRNA-135a inhibits cancer cell proliferation by targeting the c-MYC oncogene in renal cell carcinoma. Cancer Sci 104: 304-312, 2013. 